\title{
La mission des Pères Capucins auprès des Indiens de Ristigouche
}

\section{Claude Allard}

Volume 46, 1979

URI : https://id.erudit.org/iderudit/1007117ar

DOI : https://doi.org/10.7202/1007117ar

Aller au sommaire du numéro

Éditeur(s)

Les Éditions Historia Ecclesiæ Catholicæ Canadensis Inc.

ISSN

0318-6172 (imprimé)

1927-7067 (numérique)

Découvrir la revue

Citer cet article

Allard, C. (1979). La mission des Pères Capucins auprès des Indiens de Ristigouche. Sessions d'étude - Société canadienne d'histoire de l'Église catholique, 46, 87-98. https://doi.org/10.7202/1007117ar

Tous droits réservés ㄷ Les Éditions Historia Ecclesiæ Catholicæ Canadensis Inc., 1979
Ce document est protégé par la loi sur le droit d'auteur. L'utilisation des services d'Érudit (y compris la reproduction) est assujettie à sa politique d'utilisation que vous pouvez consulter en ligne.

https://apropos.erudit.org/fr/usagers/politique-dutilisation/ 


\section{La mission des Pères Capucins auprès des Indiens de Ristigouche}

Quand on passe à Ristigouche, au diocèse de Gaspé, on peut s'accorder le plaisir de jeter un coup d'œil sur la place de l'église. On y remarque une sculpture de groupe qui représente le baptême du vieux chef Micmac Membertou en 1610, baptisć à Port-Royal (Annapolis en Nouvelle-Écosse actuelle), par l'abbé Jessé Fléché. $\mathrm{Ce}$ vieux Membertou symbolise l'histoire de ce peuple dont fait partie le groupe de Micmacs de Ristigouche.

Selon le Père Pacifique, la tribu des Micmacs appartient à la grande famille des Algonquins, qui occupait jadis la moitié de l'Amérique du Nord '. Plus tard, le pays fut partagé en trois provinces ou régions, la troisième province étant Migmagig. i.e.. contrée de l'amitié ou «pleine d'agrément», tout à l'est sur les bords du grand Lac Salé, l'Extrême-Orient des Algonquins. Ils ont toujours occupé la partie orientale du Canada, comme ils disent : «la pointe extrême du Soleil Levant». Au début de notre siècle, ils étaient disséminés un peu partout en petits groupes dont Ristigouche était l'équivalent de la métropole, mais le grand chef était au Cap-Breton, avec des groupes à Halifax, Yarmouth, Pictou, Memramcook, Miramichi, Bonaventure et Gaspé. Mais on en retrouvait jusqu'à Rivière-du-Loup et Tadoussac.

Dans ce court travail, nous rappellerons brièvement le ministère pastoral des missionnaires auprès d'eux, notamment le travail des Récollets, branche de religieux franciscains à laquelle se rattache celle des Capucins. Nous verrons comment des témoins ont évalué la physionomie sociale et psychologique de ce groupe ethnique. Notre dernière partie fera état de quelques difficultés avec les Blancs, qui ont abouti au départ des Blancs de la Réserve Indienne. Toute notre documentation vient des archives de l'Évêché de Gaspé.

1 F. Pacifique, "La tribu des Micmacs», Bulletin de la Société de Géographie de Québec, vol. XVIII, 1924, pp. 65-84, auquel sont empruntés les traits qui suivent. 
De 1621 à 1648 , on voit se dérouler le travail des missionnaires récollets et jésuites auprès des Indiens Micmacs - le Père Sébastien, récollet, le Père Charles Turgis, S.J., le Père Charles du Marché, S.J. - qui rayonnent de Miscou. En 1642, le Père Richard se trouve à la vieille maison de Tchigouk (Ristigouche). Il découvre une croix dressée devant ce qu'il décrit dans le langage des Micmacs comme la «cabane de la prière». En 1644. le chef Nepsuget reçoit le baptême. Trois ans plus tard, 40 indiens reçoivent le baptême à la première chapelle. En 1648, les Récollets succèdent au Père Richard, et peu d'années après, le Père Christian Leclerc écrit que presque tous les indiens de Tchigouk sont baptisés et catholiques. De fait, en 1659 , quand $\mathrm{M}^{\mathrm{*} r}$ de Laval arrêta à Pcrcé en allant prendre possession de son siège épiscopal à Québec, il confirma un groupe imposant de personnes, dont 75 indiens de Tchigouk avec 70 blancs.

C'est une figure intéressante parmi d'autres que ce Père Chrétien Le Clercq, auteur de la Nouvelle Relation de la Gaspésie. À partir de 1676, comme missionnaire, il passe pour avoir utilisé, le premier. les hiéroglyphes pour l'enseignement de la foi. Ces signes symboliques serviront non seulement à Ristigouche mais dans le territoire qui constitue le Nouveau-Brunswick et la Nouvelle-Écosse actuels. Le Père Maillard les reprendra plus tard et aussi le Père Pacifique. à partir de 1894 .

Le Père Moreau remplace le Père Le Clercq. en 1678, au moment où le recensement donne pour Ristigouche 60 familles. comprenant 400 âmes avec trois familles françaises avec une maison et un magasin, la propriété de Richard Denys. Les années qui suivent connaissent la houle des grandes disputes France-Angleterre jusqu'à la conclusion de la guerre de Sept Ans, après la bataille des Plaines d'Abraham en 1759 et le traité de Versailles en 1763.

Entre-temps, les Récollets tiennent le coup auprès des Indiens :

Claude Moreau 1688

Michel Brûlé 1707-1720

Gelase de l'Estage 1720-1723-43

Le Père Luc 1730-1745

Le Père Ambroise 1740-1755

Et le Père Étienne, qui signe les actes du registre de 1759. 
En 1755 , les choses vont très mal pour la France en Acadie. Les Anglais y sont maîtres, c'est l'année de la dispersion des Acadiens. Les Indiens jusqu'alors installés sur l'autre rive, à l'emplacement d'Atholville à une dizaine de kilomètres de Campbellton, N.-B., décident de traverser la rivière et de se mettre sous la protection du Fort Français du Ristigouche actuel. Le Père Étienne, qui rédigea les premiers actes de 1759, fut remplacé par le Père La Brosse, S.J.. Par la suite. c'est le clergé séculier qui entra pour ainsi dire sur la ligne de feu spirituelle auprès des Indiens. Il y sera jusqu'à l'été 1894 , environ 120 ans.

Les prêtres séculiers se sont donc relayés à Ristigouche de 1772 à 1894, soit comme missionnaires-desservants, soit comme résidents. L'abbé Théod. Smith, le dernier en 1894, fut assez heureux de partir vu que son église avait brûlé. Les démarches amorcées quelques mois plus tôt auprès des Capucins, par l'intermédiaire de leur maison d'Ottawa, ont produit du fruit ; l'Évêque de Rimouski reçut l'avis officiel d'acceptation au printemps 1894 , et au mois d'août arrivent les premiers Capucins, dont le remarquable Père Pacifique de Valigny, jeune religieux de 31 ans, plein de feu, tonitruant de la voix, mais capable d'une douceur angélique. La communauté s'organisa en un couvent florissant, avec des Pères et des Frères de haute qualité et souvent pittoresques. Si on met en évidence ce Père Pacifique, c'est d'une part à cause de sa stabilité à Ristigouche, où il est mort en septembre 1943, mais surtout, d'autre part, à cause de l'œuvre intellectuelle et pastorale qu'il y a élaborée. Moins de deux mois après son arrivée, il écrit à l'Évêque de Rimouski : "J'ai déjà mon petit dictionnaire Micmac pour les confessions. Je commencerai bientôt à faire les questions moi-même sans interprète ».

Le Père Pacifique s'est mis à l'étude de tout ce qui touchait les Indiens. Il a surtout consacré des efforts fructueux à l'étude de la langue micmac, et il a rédigé plusieurs œuvres en cette langue : textes de liturgie, catéchismes, livres de prières. Par sa connaissance de la langue, il a ouvert la voie aux Sœurs du Saint-Rosaire et a ainsi facilité un travail scolaire extrêmement précieux de 1903 à 1972. Il a alimenté l'intérêt considérable que les milieux savants et politiques ont porté aux Indiens Micmacs depuis le début du siècle.

Mais ici nous sommes à un carrefour. Nous pourrions pousser plus à fond l'étude de l'œuvre du Père Pacifique, son rôle dans 
l'éveil de la fierté indienne autour de leur culture et des témoins matériels de leur personnalité collective. Mais ne vaut-il pas mieux regarder directement ce peuple, les symptômes de ses malaises face aux blancs, son goût d'indépendance qui s'est mis subitement à gronder durant les dernières années? Même s'il faut le faire de façon superficielle, prenons cette dernière orientation.

Que sont-ils, réellement, ces Micmacs? En 1924, dans le Bulletin de la Société de Géographie de Québec, le Père Pacifique leur reconnaitra trois caractéristiques :

1) «Cette tribu ne dégénère pas. Les autres diminuent graduellement; plusieurs sont éteintes ou menacent de disparaitre, tandis que celle-ci est aussi nombreuse, aussi vivace que jamais (...). La tribu des Micmacs n'a jamais été nombreuse, elle n'augmente pas. mais elle se maintient malgré les obstacles 2 ».

Et le Père Pacifique note que leur attachement à leur belle langue est une garantie de cohésion. Et il dit que quelques-uns d'entre eux écrivent bien. En plus de cette force de survivance, le Père écrit à leur sujet :

Enfin, il serait faux de prétendre qu'ils soient plus malheureux que les autres Indiens. Pourquoi le seraient-ils? Ils ont peu et ils se contentent de peu. S'ils avaient beaucoup, ils gaspilleraient beaucoup. Ils ne travaillent guère pour mettre de côté : c'est pourquoi les mauvaises saisons, la maladie et les accidents les surprennent toujours. mais quand ils sont capables. ils trouvent aisément de l'ouvrage, malgré leur inconstance naturelle. Ils sont patients dans les revers, rarement on les voit tristes. Il est vrai que les blancs ne leur ont pas toujours rendu justice : c'est qu'il n'est réellement pas aisé de traiter avec eux : il faut un mélange de douceur et de fermeté, de ménagements et d'autorité, qui se rencontre rarement. Il y a chez eux une apathie et un orgueil qui vous déconcertent ou vous irritent. Il en résulte qu'on se rend aisément coupable d'injustice à leur égard. Leur imprévoyance permet aussi de leur arracher, à peu de frais, un consentement à l'aliénation de leurs droits et de leurs propriétés, qu'ils regrettent après coup en en reconnaissant la folie ${ }^{3}$.

2) Un autre trait, écrit le Père Pacifique, c'est leur nature paisible et bienveillante, surtout (historiquement) à l'égard des Français

2 Ibid., pp. 68-70.

3 Ibid.. pp. 71-72. 
dont ils avaient reçu la foi chrétienne. Avec les Anglais, les contacts ont été plus difficiles, surtout que les Indiens se sont plaints à plusieurs reprises d'avoir été traités avec injustice par eux.

3 ) Et le dernier trait cité par le Père Pacifique, c'est leur attachement à la foi catholique. Une fois apparemment de bonne qualité théologique avec un aspect intéressant : pendant longtemps une répugnance très forte à l'égard de ce que nous pouvons appeler les mauvais esprits. Ce qui est du royaume de Satan leur fait peur, ce qui pouvait laisser croire à une place importante de cette attitude de crainte dans leur ancienne religion. À tout hasard, selon le Père Pacifique, la pire injure que vous pouviez faire à un Indien, c'était de l'envoyer au diablc. Ft un Indien de Ristigouche lui dit qu'avant de se réconcilier avec son père qui l'avait maudit de la sorte, il attendait que le vieillard le retire de là où il l'avait envoyé. Apparemment, cela devait correspondre à ce «go to hell», expression que nous avons vidée de son contenu menaçant.

À la fin du siècle dernier, un document non signé et non daté, conservé aux archives, plaide en faveur des écoles. On y découvre les traits sociaux de l'Indien de Ristigouche comme ils apparaissent aux yeux d'un observatcur attentif. Voici un large extrait de ce manuscrit intitulé Essai ${ }^{4}$.

Sujet: Déterminer à Ottawa, le Département des sauvages à construire à Ristigouche, un logement pour des religicuses qui feront l'école aux enfants micmacs. Ce département est dirigé par des protestants, peut-être francs-maçons, et partant hostiles à l'enseignement et à l'éducation catholiques.

À l'embouchure de la Rivière Ristigouche, sur sil rive gauche. se trouve un village de Micmacs renfermant quatre-vingt-six maisons, et un peu plus d'une centaine de familles. D'après les recensements, la population y est stationnaire: en 1881 soit un nombre de 475 âmes et en 1891 - 472 âmes. Ce village forme aujourd'hui le groupe de Micmacs le plus considérable de l'ancienne nation souriquoise ou micmaque qui occupait autrefois éparse çà et là l'Acadie, le Cap-Breton, le Nouveau-Brunswick et Terre-Neuve. Ce peuple a toute une tradition, des coutumes, une langue harmonieuse, d'une concision et d'une richesse étonnante (...). C'est une cuvre de haute philanthropie chrétienne que de développer la civilisation chez cette tribu qui remonte aux temps inconnus de

4 Archives de l'Évêché de Gaspé. 
l'Amérique, et de la conserver comme un monument pour les âges futurs $(\ldots)$.

Y développer la civilisation : cette tâche ne paraît pas difficile si l'on considère les progrès accomplis depuis un demisiècle. Alors c'étaient pour les demeures de ces sauvages, des cabanes en écorce de bouleaux; pour vêtements, des peaux d'animaux et surtout des couvertes en laine blanche portant encore des lisières rouges ou bleues: pour nourriture, outre le poisson, la viande des bêtes de la forêt. Et aujourd'hui on voit à la place des cabanes d'écorce qui n'existent plus, on voit de jolies maisonnettes dont plusieurs à l'apparence aiséc avec des divisions même en plâtre à l'intérieur: demeures proprement tenues qui souvent soutiendraient comparaison avantageuse avec celles de beaucoup de blancs. On dit que la gente incivile n'y vit plus, excepté peut-être dans une couple de familles par respect, je suppose, pour les antiques traditions. Ils portent avec démarche fière le costume des blancs à la coupe élégante. Presque tous cultivent un peu: plusieurs récoltent assez pour une subsistance de l'année : un micmac a vendu en mai dernier cent-cinquante minots de patates recueillies sur sa terre. On y voit des chevaux. de bonnes voitures, des vaches et jusquä des faucheuses.

Un étranger pourrait se figurer au mot samrages, que ce sont des êtres très barbares, ignorant la politesse. le savoir-vivre. l'urbanité. Que cet étranger vienne les voir à Ristigouche, il sera surpris d'y trouver des maisons où le maître le recevra dans son salon, comparativement bien monté. avec autant d'égard, et même plus que ne le feraient beaucoup de blancs. II sera surpris de voir ces sauvages suivre une conversation avec bon ton, avec l'aisance d'une personne d'une moyenne instruction. Il observera peut-être des filles micmaques qu'on pourrait appeler vraies demoiselles aussi bien que les belles québécoises. Il constatera avec plaisir cette grande douceur sympathique qui forme le trait saillant dans le caractère de cette nation.

Et par un sentiment de justice. il se dira: Quelle heureuse transformation due à l'esprit du christianisme. due aux dévouements des missionnaires.

Ces aperçus donnent à croire que les relations pouvaient être bonnes entre les deux communautés, indienne et blanche. Sans doute ; mais sous la surface, des malaises couvent. Mais qu'y a-t-il donc qui fasse mal? 
D'abord des raisons de se plaindre. Ainsi, en 1786, le gouvernement avait demandé aux Micmacs de renoncer à leur terre de chasse en faveur des blancs, terre -qui s'étendait jusqu'à la Rivière Nouvelle. La générosité du roi d'Angleterre devait assurer une légitime compensation. Et par la seule expression de ce désir, les terres furent considérées comme abandonnées et les dédommagements promis ne furent distribués que très rarement et à titre de faveur. En 1824, on rétrécit leur réserve, d'une façon exagérée aux yeux des Indiens ; 80 ans de réclamation amenèrent une rectification de ligne en 1905, au goût des blancs leurs maîtres et non des Indiens eux-mêmes. Par deux fois au cours du XIX siècle, les Indiens ont menacé de retirer leur tomahawk qu'ils avaient, selon leur expression, jeté au fond de l'eau avec la hache de guerre, dans leur soumission aux Anglais de la Nouvelle-Écosse (à la suite de l'intervention de l'abbé Bourg). Les missionnaires Faucher et Saucier (1859-69) ont empêché qu'il n'y eût quelques têtes de blancs fracassées.

Du côté des Blancs, malgré un désir évident de se rapprocher et malgré une charité facile à identifier surtout chez les missionnaires, il semble que se soit caché et se cache encore un subtil. sournois, profond, indéracinable complexe de supériorité. J'en donne un symptôme. En 1823, l'abbé Charles Painchaud écrit à M"r Plessis au sujet du choix d'un chef.Et il conclut sa lettre : «Excusez mon papier, je n'en ai point d'autre pour le moment, et la liberté que je prends de vous déranger pour des sauvages. J'ai l'honneur d'être. de $\mathrm{M}^{\mathrm{w}}$, le très humble serviteur...».

Ces choses-là se sentent et survivent aux années. Il suffit de petites étincelles pour mettre le feu. Ainsi en 1909, les Indiens écrivent à $\mathbf{M}^{* r}$ Blais, évêque de Rimouski, diocèse auquel ils appartiennent encore, et se plaignent de la séparation des Blancs et des Indiens dans leur église nouvelle. Et ils ajoutent qu'il y a là trop de Pères (ils les appellent les clergymen). À leurs yeux un seul suffirait et cela ferait moins de trouble et moins de frais dentretien.

$\mathrm{M}^{\mathrm{rr}}$ Blais répond en une lettre de cinq pages où il dit, en résumé, que la séparation a précisément pour but d'empêcher les Blancs d'évincer les Indiens en réservant à ces derniers un côté de l'église qu'ils seront les seuls à pouvoir occuper. Quant au nombre de Pères, l'Évêque leur rappelle finement que les Pères ne vivent pas à même les apports financiers de la paroisse et que leur départ 
serait une injustice et une calamité. De temps à autre, comme ça, il y a eu des symptômes de malaise qui ont fini par devenir sérieux durant le dernier quart de siècle.

\section{Ainsi, déjà, en 1956, le Père Armand, curé, écrivait à $\mathrm{M}^{* r}$ Albini Leblanc, évêque de Gaspé :}

Que dois-je faire? Connaît-on parfaitement l'âme d'un Indien? C'est tellement différent de nous... Il y a aussi le fait qu'ils n'ont jamais d'instruction dans leur langue ... c'est vrai que tous comprennent l'anglais, mais dans quelle mesure comprennent-ils? Ils parlent tous ou à peu près l'anglais, les écoles sont complètement anglaises, les catéchismes également mais dans quelle mesure assimilent-ils, ça reste une langue étrangère... Pour la plapart de ceux qui manquent la messe. la croyance à la Communion pascale est tout ce quil leur reste de foi : n'y aurait-il pas danger d'éteindre complètement la mèche qui fume encore. Et puis il y a le grand danger, très réel de les faire passer au protestantisme... nous n'avons pas encore de "mitaine protestante" ici. mais un grand nombre de ces Indiens sont imbus d'idées protestantes (le fait n'est pas surprenant. ils sont toujours rendus au Maine, d'autres vont voir les Témoins de Jéhovah de Campbellton, un grand nombre écoutent tous les dimanches, les prêches des ministres protestants à la radio ... au lieu de venir à la messe ici). Ce danger n'est pas illusoire, le fait d'ailleurs s'est produit à Caughnawaga et à Pierreville, où ils ont maintenant une " mitaine protestante». L'an dernier, nous avons essayé de non pas refuser, mais simplement retarder l'absolution dans certains cas... Or, la chose a été prise très mal, on nous a salis de toute façon à cause de cela ... (les prêtres c'est une affaire d'argent, etc., etc.). Par contre, peut-être vaudrait-il mieux de séparer tout de suite l'ivraie du bon grain ... tant pis pour les autres. De plus, c'est très possible également qu'en leur expliquant tranquillement la chose, ils vont finir par comprendre... (les Indiens sont lents à comprendre) et qu'un certain nombre d'entre eux reviendront à la pratique normale.

Qu'est-ce qui se passe? Et qu'est-ce qui se passe dans la tête des Indiens ? Voici la traduction d'une lettre ouverte adressée au journal The Tribune le 6 octobre 1971. Elle arrive après quelques troubles à la Réserve. Elle est de William Wysote, alors chef de la Bande de Ristigouche :

Cette lettre ne veut pas nous excuser au sujet des événements qui se sont passés à la Réserve durant les derniers mois. Mais 
il s'agit de voir si la cause peut se justifier grâce aux faits que tous pourront examiner.

Je me souviens, quand nous allions à l'école du jour pour les Indiens ici à Ristigouche. Il y avait une école française voisine et une clôture nous séparait. Les deux écoles étaient sous la direction des Sœurs de la même communauté. Elles nous rappelaient nos manières sauvages et la supériorité des nonIndiens ou des francophones. Même l'église était divisée : d'un côté s'assoyaient les Indiens et de l'autre les non-Indiens ; les enfants de chœur étaient aussi divisés. En vieillissant, nous en tirâmes la conclusion que les Français étaient meilleurs que nous. Comme être humain, une personne commence à éprouver du ressentiment devant une telle attitude. Tu te demandes: mais qui sont-ils d'abord? Nous sommes punis pour faire de mauvaises choses et ils s'en tirent en faisant les mêmes choses.

Dans ce temps-là il y avait des lois interdisant aux Indiens d'obtenir de l'alcool à la Commission des Liqueurs. Malgré tout ils en avaient grâce à un non-Indien. De la façon dont la loi était faite et d'après les personnes soi-disant bien éduquées qui avaient fait la loi, les Indiens ne pouvaient pas avoir d'alcool. En tout cas, je n'ai pas vu d'Indien qui le pouvait. À tout hasard, c'était la loi dans le temps, pour ne pas mentionner que c'était les non-Indiens qui avaient inventé l'eau de feu. Et c'était correct pour les Blancs de boire et pas pour les Indiens.

Personnellement, j'avais le sentiment que nous étions mauvais et que l'homme blanc nous faisait des faveurs pour notre bien. Ce fut différent à mesure que je grandis. Je continuai à l'école des Indiens jusqu'au Grade 6, équivalent du Grade 4 de nos jours. Et c'était le plus loin que je pouvais aller. $\mathrm{Si}$ vous étiez un gentil garçon ou de belle apparence, vous pouviez faire vos classes. Je n'étais ni l'un ni l'autre. Alors j'ai pris une scie à chaîne et j'ai commencé à travailler pour vivre. J'ai travaillé autour des États-Unis comme manœuvre. J'ai réalisé que tout le monde ne pensait pas que c'était mauvais d'être Indien mais plutôt que j'étais une personne qui avait des privilèges spéciaux parce que nous avions perdu notre pays aux mains des non-Indiens, par fraude et par n'importe quel moyen croche utilisé dans le temps. Cela m'aida à étudier et à trouver par moi-même des réponses aux questions qui me dérangeaient depuis si longtemps et à aider mon peuple. 
Étais-je réellement sauvage? Je me demandais: un nonIndien est-il une personne meilleure que moi? Si non, pourquoi le pense-t-il ?

Quand j'ai lu l'histoire de l'Europe. mes cheveux se sont dressés quand j’ai vu comment les pays soi-disant civilisés étaient cruels et sans cœur envers ceux qu'ils étiquetaient comme ignorants et comment ils s'en sont emparés 5.

Et le chef continue en expliquant comment lui et des membres de la Bande se sont mis à investiguer et à réagir tant auprès du gouvernement que des autres institutions. On n’a pas à évaluer l'objectivité des réactions de William Wysote mais à constater qu'il $y$ a là un potentiel affectif explosif.

En octobre 1971, le Père Donat B. Lemire, curé, écrivait à $\mathrm{M}^{\mathrm{mr}}$ Gilles Ouellet, évêque de Gaspé :

Et l'ÉGLISE : les rumeurs voulaient qu'elle fusse brûlée le 28 septembre... et ces rumeurs ont fait boule de neige ici et dans la région. Nos gens de Pointe-à-la-Croix qui demandaient une école dans leur secteur, demandent actuellement une ÉGLISE dans leur secteur. C'est une pénible mentalité de contestataires que nous affrontons. On ne veut plus venir à la messe à Ristigouche, on se fait briser la voiture, on a peur des bombes, etc., et cependant on y vient pour autre chose. Je ne vois pas la moitié de mes gens à l'église depuis plus d'un mois. On me dit que certains sont à signer une pétition qui vous sera envoyée naturellement au sujet d'une église chez eux. (Un gardien indien surveille les abords de l'église le dimanche et fait placer les voitures...) Devant cette confusion, j'ai remis la visite paroissiale en novembre. Si le climat de cette tension existe cncorc à ce moment-là, il se peut que je la remette à l'hiver tout simplement.

Chez les Indiens, il semble que le calme soit revenu, même si un sentiment de gêne plane sur les rapports. Ceux qui ne pensent pas comme le chef, sont fortement embarrassés.

Je vous envoie une découpure du Journal The Tribune où le chef s'exprime. Il y a du vrai, du moins vrai et du faux ${ }^{6}$.

5 Archives de l'Évêché de Gaspé. Dossier Ristigouche.

6 Dossier de l'Évêché de Gaspé. Dossier Ristigouche. Correspondance. 
Le 11 novembre 1971, $\mathrm{M}^{\mathrm{gr}}$ Ouellet lui répondait dans une lettre dont voici un élément important :

Je tiens à vous réitérer ma satisfaction pour le travail que vous accomplissez, mais je réalise de plus en plus qu'il est absolument nécessaire de séparer les communautés indiennes et blanches de la paroisse, parce que c'est un tour de force qui vous divise contre vous-même et rend excessivement pénible votre action pastorale.

[...]

Je vous serai reconnaissant de me tenir au courant de tout autre développement concernant la situation de la paroisse. cituation qui à la fois me préoccupe et me donne de nouveaux espoirs parce que nous avons été acculés au mur et que nous sommes obligés de repenser notre action pastorale à Ristigouche dans le but de l'améliorer. Nous avons franchi le cap du statu quo et nous sommes en route, je l'espère, vers une nouvelle forme d'action pastorale qui convienne mieux à la situation actuelle ${ }^{7}$.

Finalement, en 1975, les Pères Capucins passèrent à la corporation épiscopale tous les biens matériels administrés par la communauté. Les Blancs de Ristigouche eurent alors leur propre paroisse à Sainte-Hélènc-de-Pointe-à-la-Croix, adjacente au territoire de la Réserve, tandis que les Indiens eurent leur service religieux à l'église de Sainte-Anne-de-Ristigouche. Au plan religieux, c'est le dernier tournant de l'histoire des Indiens et des Capucins à Ristigouche.

À la fin de l'année 1979, toutes les propriétés des Blancs ont été achetées par le Gouvernement Fédéral pour être remises au territoire de la Réserve. Avant le départ des derniers Blancs, la paroisse comptait 267 foyers pour un total de 1358 baptêmes, dont 1337 de langue anglaise. Le sentiment du Père Pacifique se justifie : ce groupement montre des signes évidents de vitalité et sa natalité surprend. Mais peut-être le Père avait-il sous-estimé leur capacité de rebondissement devant une frustration accumulée pendant plusieurs années.

L'histoire a préparé cet aboutissement prévisible, aidée en cela par l'évolution d'un nationalisme partagé par les autres groupes indiens du Canada. Un coup d'œil superficiel permet aussi de croire

7 Ibid. 
que les Micmacs de Ristigouche tentent de retrouver leurs racines par-delà les siècles de colonisation. S'ils y réussissent, nous serons témoins de formes de culture religicuse, artisanale et sociale auxquelles nous n'étions pas habitués.

Claude Allard,

Gaspé. 